\title{
Optimizing human fertility and survival
}

To the editor-Whenever a gene for fertility is identified ${ }^{1}$, it is bewildering to think how variants associated with impaired fertility could have spread so widely in the population despite their obvious fitness disadvantage. Impaired fertility presently affects about 1 in 7 heterosexual couples in developed countries ${ }^{2}$. To explain this paradox, we refer to evolutionary theories on longevity that assume costs of reproductive success $^{3}$. Here we present an immunogenetic explanation for how evolutionary fitness optimizes selection for fertility with selection for survival.

Reproductive success is dependent on a Th2/Tr1 immune response at the fetal-maternal interface permitting pregnancy to proceed $^{4}$. When we studied cytokine responsiveness of the innate immune system, we found that women of normal fecundity exhibit a cytokine profile that drives naive T-cells towards a $\operatorname{Th} 2 / \operatorname{Tr} 1$ phenotype (Table 1 ). The probability of normal fecundity increased more than 10-fold when the innate cytokine profile of the women was characterized by high interleukin (IL)-10 and low tumor necrosis factor (TNF)- $\alpha$ responsiveness. The cytokine profile of women with impaired fertility was characterized by low IL-10 and high TNF- $\alpha$ responsiveness.

Previous attempts to identify genetic polymorphisms that associate with different TNF- $\alpha$ responsiveness have been unsuccessful. Earlier we have identified haplotypes that segregate with IL-10 responsiveness ${ }^{5}$, as well as several novel single-nucleotide polymorphisms in the distal promoter of the gene encoding IL-10 (ref. 6). Here we present data on cytokine responsiveness in healthy subjects stratified for a nucleotide polymorphism at position -2849 of the IL-10 promoter (Fig. 1). It seems that carriers of the -2849 AA genotype have significantly lower IL-10 responsiveness upon stimulation with endotoxin. Therefore, using a large set of married women with completed families, we tested whether this genotype was enriched among women with impaired fertility. Congruent with a low IL-10 phenotype as described, the -2849 AA genotype was twofold more prevalent among 73 women who remained childless when compared to the prevalence among 323 women with normal fecundity $(13.6 \%$ and $5.6 \%$ respectively, $\left.\chi^{2}=5.9, P=0.014\right)$. This difference in genotype distribution strongly supports the notion that human fertility is depen- dent on the cytokine profile of the innate immune system.

Critical for understanding the high proportion of women with impaired fertility is the relation of the innate immune system to the outcome of infectious disease. We have previously shown that subjects with the low IL-10 and high TNF- $\alpha$ responsiveness that drives naive T-cells towards a Th1 phenotype were protected from fatal outcome of infection ${ }^{8}$. In times when half of all newborns died from infection before reaching adolescence, survivors were strongly selected for resistance to infection. We propose that during evolution selection for fertility (a cytokine profile that favors the development of Th2/Tr1-type T-cells) is optimized with selection for survival (a cytokine profile that favors the development of Th1-type T-cells). The current data also provide an immunogenetic explanation for the inverse association betw een family size and longevity of British aristocrats ${ }^{3}$ under conditions in which infection was a major cause of early death. Under those conditions, an innate cytokine profile supportive of Th1-type T-cells favored survival of infectious diseases, including cholera and tuberculosis. However, women with such a cytokine profile would have been less likely to have successful pregnancies.

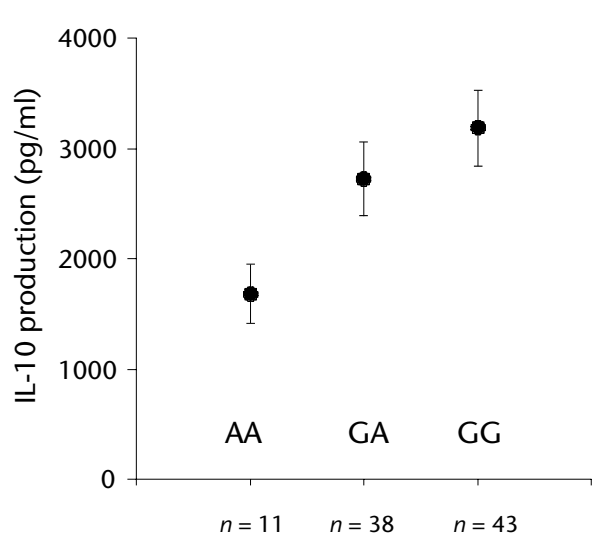

Fig. 1 IL-10 responsiveness stratified for a single nucleotide polymorphism in the promoter for the gene encoding IL-10. Data are presented as means \pm s.e.m. Production of IL-10 was determined in whole-blood samples upon stimulation with endotoxin $(1000 \mathrm{ng} / \mathrm{ml})$ for $24 \mathrm{~h}$. Sampling was performed in 92 unrelated healthy subjects and described earlier ${ }^{8}$. The single AG nucleotide polymorphism at position -2849 of the IL-10 promoter region $5^{\prime}$ to the ATG start site was determined in genomic DNA (ref. 6). IL-10 production was significantly lower in carriers of the AA genotype compared to the other genotypes, ANOVA: $P=0.021$.

Table 1 Association between reproductive success and innate cytokine responsiveness

\begin{tabular}{lllll} 
Production of IL-10 & low & low & high & high \\
Production of TNF- $\alpha$ & high & low & high & low \\
Women with normal fecundity (\#) & 1 & 11 & 14 & 6 \\
Women with impaired fertility (\#) & 8 & 13 & 10 & 3 \\
Odds ratio $(95 \% \mathrm{Cl})$ & $1(-)$ & $6.7(0.7-63)$ & $11.2(1.2-104)$ & $16(1.3-195)$ \\
\hline
\end{tabular}

Production of IL-10 (supportive of Th2/Tr1-cell development) and TNF- $\alpha$ (supportive of Th1-cell development) was determined in whole-blood samples upon stimulation with endotoxin $(1000 \mathrm{ng} / \mathrm{ml})$ for 24 and $4 \mathrm{~h}$, respectively ${ }^{8}$. The concentrations of cytokines were measured in supernatants by ELISA and dichotomized as low and high around the median. Impaired fertility was defined as having had at least 3 consecutive spontaneous abortions before $16 \mathrm{wk}$ of gestation. Women with chromosomal abnormalities, anatomical uterine defects, pro-thrombotic disorders and co-morbid conditions were excluded. $\mathrm{Cl}$, confidence interval. 